\title{
Boundary Layer Flow Over a Vertical Cylinder Embedded in a Porous Medium Moving With Non Linear Velocity
}

\author{
TAREK G. EMAM, \\ Department of Mathematics, College of Science and Arts- Khulais, university of Jeddah,Jeddah, \\ SAUDI ARABIA. \\ Department of Mathematics, Faculty of Science, Ain Shams University, Cairo, \\ EGPT.
}

\begin{abstract}
In this work, we consider a steady flow of an incompressible fluid over a cylinder which is semi-infinite. The cylinder is embedded in a porous medium and is considered to move vertically with nonlinear velocity. A system of ordinary differential equations is obtained from the partial differential equations governing the motion using a self-similarity transformation. Such system of ordinary differential equations is solved numerically after obtaining the missed initial conditions. The problem has been solved analytically for the linear velocity case. Numerical and analytical results for such case are given to validate the numerical method used.

Solution. Keywords: Boundary Layer Flow, Porous Medium, Moving Cylinder, Nonlinear Velocity, Similarity
\end{abstract}

Received: August 14, 2020. Revised: December 13, 2021. Accepted: December 27, 2021. Published: December 31, 2021.

\section{Introduction}

Many applications in industry and engineering arise from the flow of Newtonian and Non-Newtonian fluids. Authors have been interested in such fields for the last few decades. The problem of boundary layer flow over a cylinder, which is our concern in this work, has many applications such as glass fiber production, the drawing of wires, as well as plastic and metallurgy industries. Sakiadis [1] was the first to study boundary layer flow behavior on a cylinder moving in a Newtonian fluid. He obtained a numerical solution using a similarity transformation. Rotte and Beek have given Some models for the calculation of heat transfer coefficients to a moving continuous cylinder[2]. Ganesan and Loganathan [3] have introduced the problem of radiation and mass transfer effects on flow of an incompressible viscous fluid past a moving vertical cylinder. Recently Ado-Eldahab and Salem [4] have studied the flow and heat transfer of non-Newtonian powerlaw fluid with diffusion and chemical reaction on a moving cylinder. Amkadni and Azzouzi [5] have analyzed the steady flow of an incompressible electrically conducting fluid over a semi-infinite moving vertical cylinder in the presence of a uniform transverse magnetic field. Elbashbeshy et al [6] have analyzed the problem of boundary layer flow over a stretching horizontal cylinder embedded in a porous medium. They have considered the effects of thermal radiation, heat transfer, and suction/injection. Abdul Rehman et al [7] have given an analytic solution to the problem of, axisymmetric Stagnation Flow of a Micropolar Fluid in a Moving Cylinder. Haroon et al [8] have presented an investigation provides an view in the steady, incompressible and electrically conducting boundary layer flow of viscoelastic nanofluid flowing due to a moving, linearly stretched surface. In this paper we present a solution to the problem of a vertically moving cylinder with nonlinear velocity. Analytic solution is given for the case of linear velocity and a comparison between analytic and numerical solutions is given in some cases to validate the numerical method used in this paper.

\section{Mathematical Formulation}

Consider a steady incompressible laminar flow past a moving cylinder. The cylinder is assumed to be semi-infinite and vertical with radius $R$. A uniform transverse magnetic filed with strength $B_{0}$ is applied. We also assume that the fluid properties are constant. The induced magnetic field is neglected since we consider that the Reynolds number is very small. Along the axis of the cylinder we measure the axial coordinate $x$ while the radial coordinate $r$ is measured normal to the axis of the cylinder. The external velocity is taken in the form $u_{e}(x)=u_{\infty}\left(\frac{x}{l}\right)^{n}$, where $u_{\infty}>0$. Such assumptions along with the boundary layer approximation results in the following governing equations:

$$
\begin{aligned}
& \frac{\partial r u}{\partial x}+\frac{\partial r v}{\partial r}=0 \\
& u \frac{\partial u}{\partial x}+v \frac{\partial u}{\partial r}=\frac{\nu}{r} \frac{\partial}{\partial r}\left(r \frac{\partial u}{\partial r}\right)+u_{e} \frac{d u_{e}}{d x}+\frac{\nu}{\kappa_{p}}\left(u_{e}-u\right)
\end{aligned}
$$

subject to the conditions:

$$
u(R, x)=u_{w}\left(\frac{x}{l}\right)^{n}, v(R, x)=0, \lim _{r \rightarrow \infty} u(r, x)=u_{e}\left(\frac{x}{l}\right)
$$

where $u$ and $v$ are the velocity components along the directions of $x$ and $r$ respectively, $\nu$ is the kinematic viscosity, $\rho$ is the fluid density, and $\sigma$ is the electrical conductivity of the fluid, $l$ is the characteristic length

and $\kappa_{p}$ is the porosity of the medium. We define the stream function $\psi$ as

$$
r u=\frac{\partial \psi}{\partial r}, \quad r v=-\frac{\partial \psi}{\partial x}
$$


Substituting from equation (4) into equations (1) and (2) we find that equation (1) is satisfied identically and equation (2) takes the form:

$$
\begin{gathered}
\frac{1}{r^{2}} \frac{\partial \psi}{\partial r} \frac{\partial^{2} \psi}{\partial x \partial r}+\frac{1}{r^{3}} \frac{\partial \psi}{\partial x} \frac{\partial \psi}{\partial r}-\frac{1}{r^{2}} \frac{\partial \psi}{\partial x} \frac{\partial^{2} \psi}{\partial r^{2}}=\frac{\nu}{r^{3}} \frac{\partial \psi}{\partial r} \\
-\frac{\nu}{r^{2}} \frac{\partial^{2} \psi}{\partial r^{2}}+\frac{\nu}{r} \frac{\partial^{3} \psi}{\partial r^{3}}+u_{e} \frac{d u_{e}}{d x}+\frac{\nu}{\kappa_{p}}\left(u_{e}-\frac{1}{r} \frac{\partial \psi}{\partial r}\right)
\end{gathered}
$$

and the conditions (3) are transformed into the form:

$$
\begin{array}{r}
\frac{\partial \psi(R, x)}{\partial r}=R u_{w}\left(\frac{x}{l}\right)^{n}, \frac{\partial \psi(R, x)}{\partial x}=0, \\
\lim _{r \rightarrow \infty}\left(\frac{1}{r} \frac{\partial \psi(r, x)}{\partial r}\right)=u_{\infty}\left(\frac{x}{l}\right)^{n}
\end{array}
$$

we look for a solution in the form

$$
\begin{gathered}
\psi(r, x)=\sqrt{\frac{\nu u_{\infty} R(n+1)}{2}\left(\frac{x}{l}\right)^{n+1}} R f(\eta), \\
\eta=\sqrt{\frac{u_{\infty}}{2 \nu(n+1) R}\left(\frac{x}{l}\right)^{n-1}} \frac{1}{R}\left(r^{2}-R^{2}\right)
\end{gathered}
$$

where $f$ is the dimensionless stream function and $\eta$ is the similarity variable. Upon substituting in equations (5) and (6), we get:

$$
\begin{aligned}
& \quad \frac{2}{n+1}(\eta K+1) f^{\prime \prime \prime}(\eta)+\left(\frac{2 K}{n+1}+\frac{\epsilon(n+1)}{2} f(\eta)\right) f^{\prime \prime}(\eta) \\
& +\quad n \epsilon\left(1-f^{\prime 2}(\eta)\right)-\kappa\left(f^{\prime}(\eta)-1\right)=0 \\
& f(0)=0, \quad f^{\prime}(0)=\frac{u_{w}}{u_{\infty}}=a, \quad f^{\prime}(\infty)=1
\end{aligned}
$$

where $K=\sqrt{\frac{\nu(n+1)}{u_{\infty} R}\left(\frac{l}{x}\right)^{n-1}}, \epsilon=\frac{R}{l}$, and the permeability parameter is $\kappa=\frac{R \nu}{\kappa_{p} u_{\infty}}\left(\frac{l}{x}\right)^{n-1}$.

The system $(8)-(9)$ is transformed into a system of first order differential equations through assuming that: $y_{1}=f, y_{2}=f^{\prime}$, $y_{3}=f^{\prime \prime}$ to get

$$
\begin{aligned}
& y_{1}^{\prime}(\eta)=y_{2}(\eta) \\
& y_{2}^{\prime}(\eta)=y_{3}(\eta) \\
& y_{3}^{\prime}(\eta)=y_{2}(\eta) \\
& \quad \frac{2}{n+1}(K \eta+R) y_{3}^{\prime \prime}(\eta)=-\left(\frac{2 K}{n+1}+\frac{\epsilon(n+1)}{2} y_{1}(\eta)\right) \\
& \times \quad y_{2}(\eta)-n \epsilon\left(1-\left(y_{2}^{2}(\eta)\right)+\kappa\left(y_{2}(\eta)-1\right)\right.
\end{aligned}
$$

subject to the initial conditions:

$$
y_{1}(0)=0, \quad y_{2}(0)=a, \quad y_{3}(0)=s
$$

Suitable numerical values are given for $a, n, \kappa, \epsilon$, and $K$. the value of $s$ is priori unknown that is determined as part of the solution. We use Mathematica to define a function $F[s]=$ $N D$ Solve $[(10)-(14)]$. The value of $s$ is obtained through solving the equation $y_{1}\left(\eta_{\max }\right)=1$. A suitable start value of $\eta$ is taken and then increased to reach $\eta_{\max }$ for which the difference between two successive values of $s$ is less than $10^{-7}$. So the problem now is an initial value problem which is then solved using NDSolve

\section{Results and Discussions}

\subsection{Special Case: $\mathbf{N}=1$}

Here we consider the case $n=1$ which means that the cylinder moves with linear velocity. Equation (8) takes the form

$$
(\eta K+1) f^{\prime \prime \prime}(\eta)+(K+\epsilon f(\eta)) f^{\prime \prime}(\eta)+\epsilon\left(1-f^{\prime 2}(\eta)\right)-\kappa\left(f^{\prime}(\eta)-1\right)=0
$$

subject to the conditions:

$$
f(0)=0, \quad f^{\prime}(0)=\frac{u_{w}}{u_{\infty}}=a, \quad f^{\prime}(\infty)=1
$$

where $K=\sqrt{\frac{2 \nu}{u_{\infty} R}}$, and $\kappa=\frac{R \sigma B_{0}^{2}}{\rho u_{\infty}}$.

We seek for an exact solution to the linear case in the form

$$
f(\eta)=\eta+p+q e^{-\mu \eta}
$$

where $p, q, \mu$ are constants and $\mu>0$. So

$$
f^{\prime}(\eta)=1-q \mu e^{-\mu \eta}
$$

Applying the conditions $f(0)=0, f^{\prime}(0)=a$ we find that

$$
f(\eta)=\eta+\frac{a-1}{\mu}\left(1-e^{-\mu \eta}\right)
$$

the condition $f^{\prime}(\infty)=1$ is satisfied identically which can be observed from equation (18).

Substituting equation (19) into equation (15) we get the following:

$$
\left((\eta K+1) \mu^{2}-\mu K-\eta \epsilon \mu-2 \epsilon-\kappa\right)(a-1)-\epsilon(a-1)^{2}=0
$$

Using the fact that $a \neq 1$ and equating the coefficients of $\eta$ and $\eta^{0}$, we get

$$
\mu=\epsilon \sqrt{\frac{u_{\infty} R}{2 \nu}}=\frac{\epsilon}{K}
$$

and

$$
\kappa=\left(\frac{\epsilon}{K}\right)^{2}-\epsilon(a+2)
$$

hence the exact solution of equation (15) is

$$
f(\eta)=\eta+\frac{(a-1) k}{\epsilon}\left(1-e^{-\frac{\epsilon}{K} \eta}\right)
$$

where $\kappa$ is given by equation $(22)$

\subsection{Validation of the Numerical Method}

To validate the numerical method used in this paper we compare the numerical solution with the exact one for the case $n=1$. Table 1 gives a comparison between the values of $f^{\prime \prime}(0)$ for $K=2, \epsilon=1$ and different values of $a$ Table 1:Values of $f^{\prime \prime}(0)$, where $K=0.2, \epsilon=1$

\begin{tabular}{|c|c|c|}
\hline$a$ & Exact Solution & Numerical Solution \\
\hline 1.2 & -1 & -1 \\
1.5 & -2.5 & -2.5 \\
2 & -5 & -5 \\
3 & -10 & -10 \\
\hline
\end{tabular}

The results shown in table 1 ensures that the numerical method used in this paper is valid. 


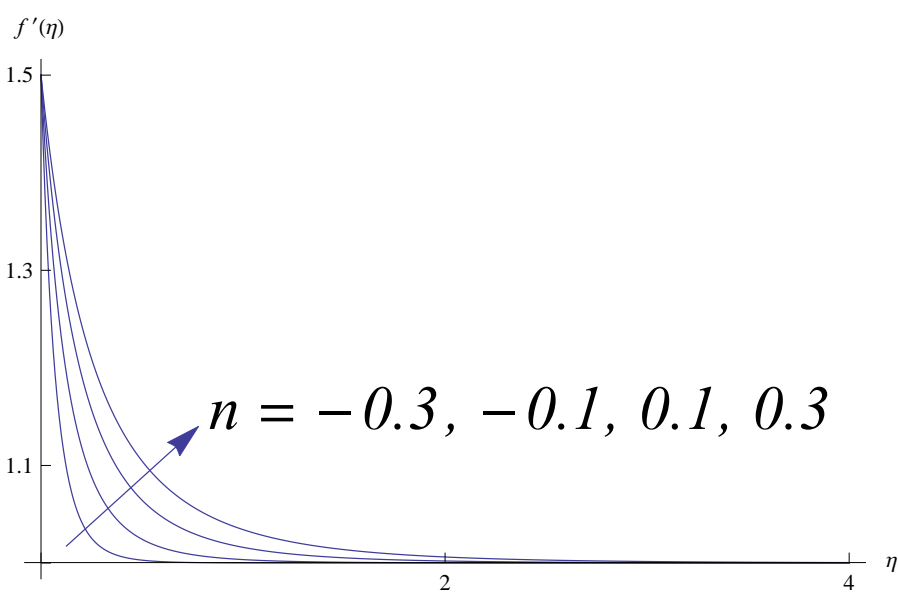

Fig. 1. Variation of the fluid velocity with the nonlinearity parameter $n$, where $K=2, a=1.5, \epsilon=1$, and $\kappa=1$.

\subsection{Case: $n \neq 1$}

Numerical solutions where $n \neq 1$ are obtained using the method described at the end of section 2 .

The variations of the velocity $f^{\prime}(\eta)$ with the similarity variable $\eta$ are plotted for different values of the considered parameters. The figures (1-4) show that the fluid velocity $f^{\prime}(\eta)$ decreases with the increase of $\eta$ till it reaches the ambient fluid velocity that is $f^{\prime}(\eta)=1$. Figure 1 shows the variation of $f^{\prime}(\eta)$ for different values of the parameter $n$. From the figure one can observe that as $n$ elevates the value of $f^{\prime}(\eta)$ increases. since as $n$ increases the cylinder velocity increases which enforces the fluid velocity to increase as shown in the figure. The variation of the velocity $f^{\prime}(\eta)$ with the permeability parameter $\kappa$ is shown in figure $2 . \kappa$ plays a considerable role in controlling the fluid velocity. The value of $\kappa_{p}$ is a measure of how it is easy to penetrate the porous medium. $\kappa$ is the reciprocal of $\kappa_{p}$ so the increase of the permeability parameter $\kappa$ increases the resistance to the fluid motion and consequently the fluid velocity $f^{\prime}(\eta)$ decreases which coincides with the results shown in figure 2. The parameter $\epsilon$ has a considerable effect on the fluid velocity as depicted in figure 3 . One can notice that the decrease of $\epsilon=\frac{R}{l}$ increases the fluid velocity. A reasonable explanation of this is that the decrease of $R$ results in decreasing the cylinder surface area, so the cylinder shrinks and as a result the space provided for the fluid free stream velocity increases. Thus the tendency of the fluid velocity to be a free stream is enhanced. Figure 4 ensures the fact that as the fluid velocity increases as the initial velocity increases.

\section{Conclusion}

An investigation of the problem of a boundary layer flow over a vertical cylinder moving with nonlinear velocity is given. An exact solution has been found for some special cases. Similarity solution of the problem has been given and the profiles of the fluid velocity have been plotted to show the variation of the fluid velocity with the considered parameters. The following results have been obtained:

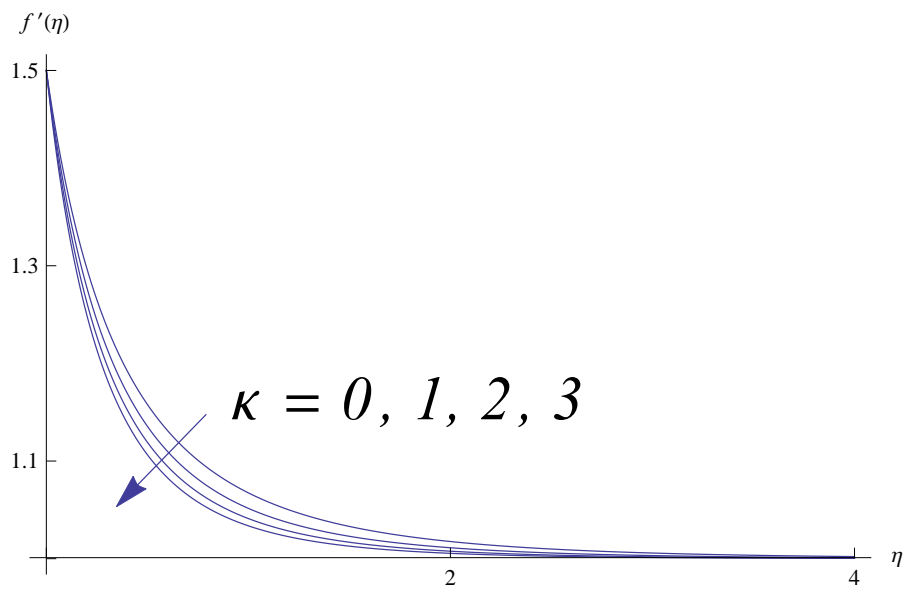

Fig. 2. Variation of the fluid velocity with the permeability parameter $\kappa$, where $K=2, a=1.5, \epsilon=1$, and $n=0.5$.

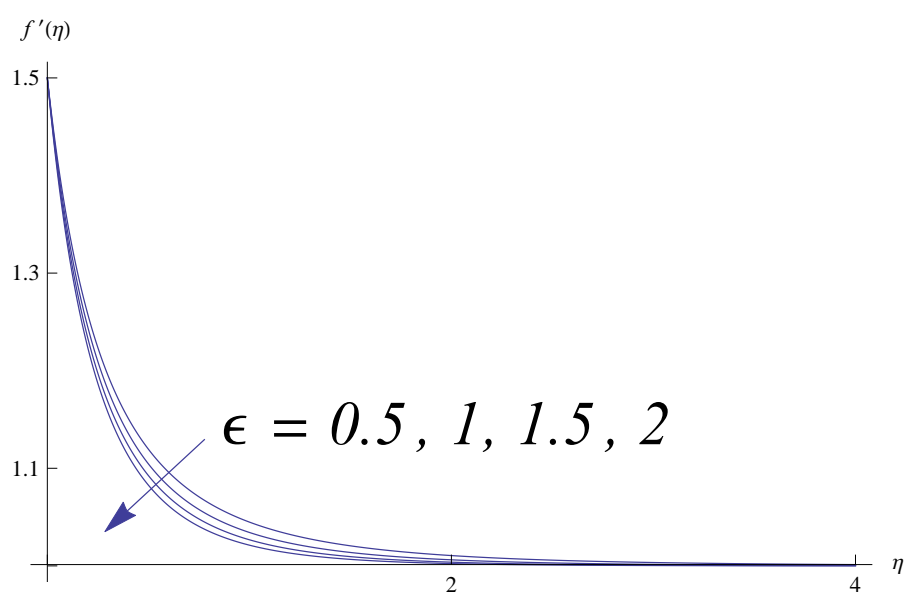

Fig. 3. Variation of the fluid velocity with the parameter $\epsilon$, where $K=2$, $a=1.5, n=0.3$, and $\kappa=1$.

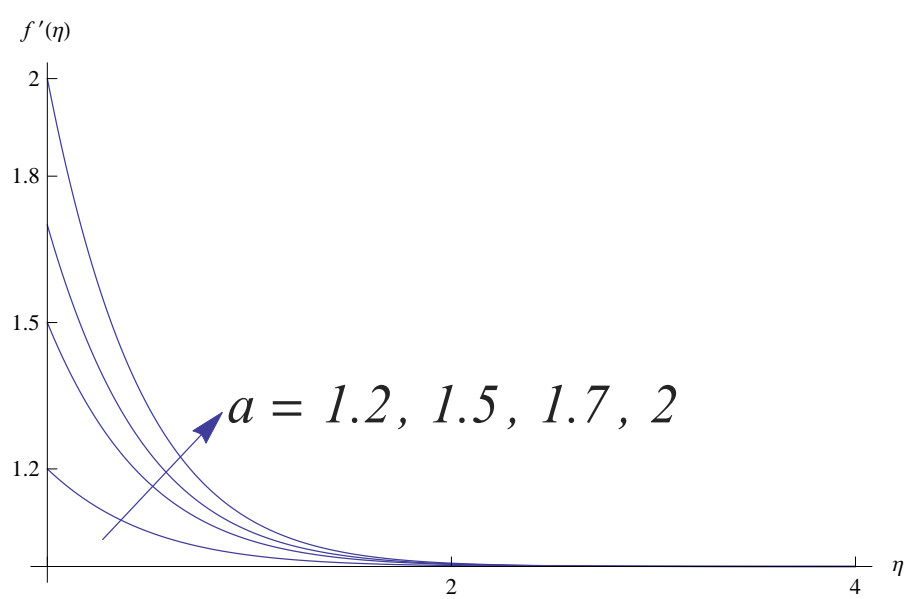

Fig. 4. Variation of the fluid velocity with the initial velocity parameter, where $K=0.2, n=0.3, \epsilon=1$, and $\kappa=1$. 
- The fluid velocity increases as $n$, the parameter of nonlinearity increases and as the fluid initial velocity increases also.

- The fluid velocity decreases with the increase of the permeability parameter as well as the cylinder radius.

\section{References}

[1] B. C. Sakiadis, Boundary-layer behavior on continuous solid surfaces: II. The boundary layer on a continuous flat surface, AIChE Journal 7(2) (1961), 221-225.

[2] J. W. Rotte and W. J. Beek, Some models for the calculation of heat transfer coefficients to a moving continuous cylinder, Chemical Engineering Science 24 (4) (1969), 705-716.

[3] P. Ganesan and P. Loganathan, Radiation and mass transfer effects on flow of an incompressible viscous fluid past a moving vertical cylinder, International Journal of Heat and Mass Transfer 45 (21) (2002), 4281-4288.

[4] E. M. Abo-Eldahab and A. M. Salem, MHD Flow and heat transfer of non-Newtonian power law fluid with diffusion and chemical reaction on a moving cylinder, Heat and Mass Transfer 41 (8) (2005), 703-708.

[5] M. Amkadni and A. Azzouzi, On a similarity solution of MHD boundary layer flow over a moving vertical cylinder, Differential Equations and Nonlinear Mechanics, Volume 2006, Article ID 52765,1-9.

[6] Elsayed M. A. Elbashbeshy, T. G. Emam, M. S. El-Azab, and K. M. Abdelgaber, Effect of thermal radiation on flow, heat, and mass transfere of a nanofluid over a stretching horizontal cylinder embedded in a porous medium with suction/injection, J. porous media, 18(3) (2015), 215-229.

[7] Abdul Rehman, Saleem Iqbal, Syed Mohsin Raza, Axisymmetric Stagnation Flow of a Micropolar Fluid in a Moving Cylinder: An Analytical Solution, Fluid Mechanics, 2 (1) (2016) 1-7.

[8] Haroon Rasheed, Abdul Rehman, Naveed Sheikh, Saleem Iqbal. MHD Boundary Layer Flow of Nanofluid over a Continuously Moving Stretching Surface. Applied and Computational Mathematics. Vol. 6 (6) (2017) 265-270.

\section{Creative Commons Attribution License 4.0 (Attribution 4.0 International, CC BY 4.0)}

This article is published under the terms of the Creative Commons Attribution License 4.0

https://creativecommons.org/licenses/by/4.0/deed.en_US 